INPLASY

PROTOCOL

To cite: Gan.

Sonothrombolysis for acute ischemic stroke: a metaanalysis of randomized controlled trials. Inplasy protocol 202160019. doi: 10.37766/inplasy2021.6.0019

Received: 07 June 2021

Published: 07 June 2021

Corresponding author: Xinling Gan

452027071@qq.com

Author Affiliation: West China Hospital, Sichuan University.

Support: NNSF:81972146.

Review Stage at time of this submission: Preliminary searches.

Conflicts of interest:

None declared.

\section{Sonothrombolysis for acute ischemic stroke: a meta-analysis of randomized controlled trials}

Gan, $\mathrm{X}^{1}$.

Review question / Objective: The aim of this meta-analysis of randomized controlled trials is to evaluate the efficacy and safety of sonothrombolysis for acute ischemic stroke. Information sources: EMBASE, Web of Science and Cochrane library databases.

Eligibility criteria: (a) randomized study design; (b) comparison of sonothrombolysis (ultrasound treatment with standard intravenous thrombolysis) with or without microbubble versus sham (inactive) ultrasound treatment with standard intravenous thrombolysis or ultrasound treatment (complication to IV) versus no ultrasound treatment (complication to IV); (c) patients with acute ischemic stroke; (d) publication in English.

INPLASY registration number: This protocol was registered with the International Platform of Registered Systematic Review and Meta-Analysis Protocols (INPLASY) on 07 June 2021 and was last updated on 07 June 2021 (registration number INPLASY202160019).

\section{INTRODUCTION}

Review question / Objective: The aim of this meta-analysis of randomized controlled trials is to evaluate the efficacy and safety of sonothrombolysis for acute ischemic stroke.
Condition being studied: 1.Ischemic stroke is the leading cause of disability and the second leading cause of death worldwide. approximately 690000 individuals in the United States experience an ischemic stroke. For those who survive, $50 \%$ have some hemiparesis, $30 \%$ are unable to walk without assistance, $26 \%$ are dependent in 
activities of daily living at 6 months after stroke, and $15 \%$ to $30 \%$ are left permanently disabled, which lead to a heavy burden on the family and society. Considering the "recanalization hypothesis", which states that reopening of occluded vessels can save threatened ischemic tissues, faster restoration of cerebral tissue blood flow associated with better neurological recovery. The early intervention to restore maximal repercussion of brain tissue is the mainstay of patient treatment and future investigation. 2.Intravenous thrombolytic therapy is an effective acute treatment option for ischemic stroke patients approved by FDA. However, its success rate is low; 3 . To increase the rate of arterial recanlization without increasing the risk of hemorrhagic complications, some researchers attempted to use the effect of energy delivered by sound waves on fibrin strands in the thrombolytic therapy, which called sonothrombolysis. Recently, a similar idea has been proposed by using ultrasound microbubble contrast agents. some published trails examining the efficacy of Sonothrombolysis with or without microbubble have shown its beneficial effects on recanalization and short-term outcomes in patients with acute Ischemic stroke, while others not.

\section{METHODS}

Participant or population: (a) randomized study design; (b) comparison of sonothrombolysis (ultrasound treatment with standard intravenous thrombolysis) with or without microbubble versus sham (inactive) ultrasound treatment with standard intravenous thrombolysis;(c) patients with acute ischemic stroke.

Intervention: Sonothrombolysis with or without microbubble.

Comparator: Intravenous thrombolysis.

Study designs to be included: Randomized controlled trials.

Eligibility criteria: (a) randomized study design; (b) comparison of sonothrombolysis (ultrasound treatment with standard intravenous thrombolysis) with or without microbubble versus sham (inactive) ultrasound treatment with standard intravenous thrombolysis or ultrasound treatment (complication to IV) versus no ultrasound treatment (complication to IV); (c) patients with acute ischemic stroke; (d) publication in English.

Information sources: EMBASE, Web of Science and Cochrane library databases.

Main outcome(s): (a) SICH rate, whereby SICH was defined as intracerebral hemorrhage confirmed via computed tomography or other imaging that was associated with an increase of $\geq 4$ points on the NIHSS; (b) all-cause mortality at 90 days; (c) recanalization rate after thrombolysis, whereby recanalization was defined as a Thrombolysis in Brain Ischemia (TIBI) classification of 4 or 5; (d) good clinical outcome at 90 days, defined by modified Rankin Scale(MRS) score $\mathbf{0 - 1}$; (e)neurological improvement, defined by changes in NIHSS scores within $24 \mathrm{~h}$ of treatment (reduction of $\geq 4$ NIHSS points compared with the baseline NIHSS score or a total NIHSS score of $\leq 3$ ).

Quality assessment / Risk of bias analysis: The quality of the selected studies was assessed by the Cochrane risk of bias tool regarding random sequence generation, allocation concealment, blinding of participants and personnel, blinding of outcome assessment, incomplete outcome data, selective reporting and other biases. The quality of each item was rated as low, unclear, or high. Two reviewers independently assessed each item, and any disagreements were resolved via discussion or by consultation with the corresponding author.

Strategy of data synthesis: In this metaanalysis, we compared sonothrombolysis with or without microbubble versus intravenous thrombolysis alone or ultrasound versus no ultrasound treatment in terms of the following outcomes: (a) $\mathrm{SICH}$ rate, whereby $\mathrm{SICH}$ was defined as intracerebral hemorrhage confirmed via 
computed tomography or other imaging that was associated with an increase of $\geq 4$ points on the NIHSS; (b) all-cause mortality at 90 days[19]; (c) recanalization rate after thrombolysis, whereby recanalization was defined as a Thrombolysis in Brain Ischemia (TIBI) classification of 4 or 5; (d) good clinical outcome at 90 days, defined by modified Rankin Scale(MRS) score $\mathbf{0 - 1}$; (e)neurological improvement, defined by changes in NIHSS scores within $24 \mathrm{~h}$ of treatment (reduction of $\geq 4$ NIHSS points compared with the baseline NIHSS score or a total NIHSS score of $\leq 3$ ).

Subgroup analysis: Stroke location (anterior or posterior circulation); Sonothrombolysis with microbubble versus Sonothrombolysis without microbubble.

Sensitivity analysis: Elimination of individual studies by Revman software.

Country(ies) involved: China.

Keywords: Sonothrombolysis; ischemic stroke; recanlization.

Contributions of each author:

Author 1 - Xinling Gan.

Email: 452027071@qq.com 